\title{
Role of surgery in a case-control study of patients with clinical stage IIIA small cell lung cancer
}

\author{
Shuangyi Li ${ }^{1 \#}$, Kaiqi Jin ${ }^{1 \#}$, Yingying Pan $^{2 \#}$, Chunxiao Wu ${ }^{3}$, Shengxiang Ren ${ }^{2}$, Gening Jiang ${ }^{1}$, Peng Zhang ${ }^{1}$ \\ ${ }^{1}$ Department of Thoracic Surgery, Tongji University Affiliated Shanghai Pulmonary Hospital, Tongji University School of Medicine, Shanghai, \\ China; ${ }^{2}$ Department of Oncology, Tongji University Affiliated Shanghai Pulmonary Hospital, Tongji University School of Medicine, Shanghai, \\ China; ${ }^{3}$ Shanghai Municipal Center for Disease Control and Prevention, Shanghai, China \\ Contributions: (I) Conception and design: P Zhang; (II) Administrative support: P Zhang; (III) Provision of study materials or patients: G Jiang, S Ren, \\ C Wu; (IV) Collection and assembly of data: S Li, K Jin, Y Pan; (V) Data analysis and interpretation: S Li, K Jin, Y Pan; (VI) Manuscript writing: All \\ authors; (VII) Final approval of manuscript: All authors. \\ "These authors contributed equally to this work. \\ Correspondence to: Peng Zhang, MD, PhD. Department of Thoracic Surgery, Tongii University Affiliated Shanghai Pulmonary Hospital. No. 507 \\ Zhengmin Road, Shanghai 200433, China. Email: zhangpeng1121@tongji.edu.cn; Gening Jiang, MD. Department of Thoracic Surgery, Tongji \\ University Affiliated Shanghai Pulmonary Hospital. No. 507 Zhengmin Road, Shanghai 200433, China. Email: jgnwp@aliyun.com; Shengxiang \\ Ren, MD. Department of Oncology, Tongji University Affiliated Shanghai Pulmonary Hospital. No. 507 Zhengmin Road, Shanghai 200433, China. \\ Email: harry_ren@126.com.
}

Background: The role of surgery in the multidisciplinary treatment of clinical stage IIIA small cell lung cancer is yet to be verified. This study was performed to determine the benefit of surgery in patients with stage IIIA small cell lung cancer.

Methods: Patients diagnosed with stage IIIA small cell lung cancer at Shanghai Pulmonary Hospital from 2005 to 2015 were included and divided into two groups: the surgery with neoadjuvant and adjuvant chemotherapy group and the concurrent chemo-radiotherapy group. Overall survival was compared between the two groups. A multivariate Cox regression model was constructed to evaluate factors associated with overall survival.

Results: Of 69 patients with stage IIIA small cell lung cancer during the study period, 40 patients (58\%) underwent surgery with neoadjuvant and adjuvant chemotherapy, and 29 patients (42\%) underwent concurrent chemo-radiotherapy. Patients in the surgery with neoadjuvant and adjuvant chemotherapy group had a longer overall survival compared with patients in the concurrent chemo-radiotherapy group (median survival: 33.1 vs. 16.2 months, respectively; 2 -year overall survival: $44.2 \%$ vs. $14.9 \%$, respectively; log-rank: $\mathrm{P}=0.045$ ). A multivariate analysis revealed that surgery with neoadjuvant and adjuvant chemotherapy (hazard ratio: 0.374 ; $95 \%$ confidence interval: $0.173-0.808, \mathrm{P}=0.012$ ) was independently associated with overall survival.

Conclusions: Patients with stage IIIA small cell lung cancer treated with surgical resection plus chemotherapy demonstrated longer overall survival compared with those who underwent concurrent chemo-radiotherapy. Surgery may be an option for clinical stage IIIA small cell lung cancer after induction chemotherapy in selected patients.

Keywords: Small cell lung cancer (SCLC); surgery; chemotherapy

Submitted Oct 09, 2020. Accepted for publication Mar 14, 2021.

doi: $10.21037 /$ jtd-20-3047

View this article at: http://dx.doi.org/10.21037/jtd-20-3047

(c) Journal of Thoracic Disease. All rights reserved. 


\section{Introduction}

Small cell lung cancer (SCLC) accounts for approximately $13 \%$ of bronchogenic carcinomas (1). According to a report from the World Health Organization, there will be 287,683 new cases of SCLC globally in 2020 (2) and 29,660 new cases in the United States (3). Compared with nonSCLC (NSCLC), SCLC has higher metastatic potential and poorer survival outcomes. For patients with limitedstage SCLC, chemo-radiotherapy has been the standard treatment since the early 1970s based on two prospective randomized clinical trials from the Medical Research Council in 1973 (4) and the Lung Cancer Study Group in 1994 (5), which demonstrated that surgery had no benefit for patients with SCLC. After 2000, with a number of retrospective studies indicating potential benefits on 5-year survival in patients with SCLC who underwent surgery (6-9), surgical treatment combined with chemotherapy is recommended in patients with limited disease without lymph node metastasis $(10,11)$. Thus, the role of surgery for SCLC is being reevaluated. However, studies reevaluating the status of surgery are retrospective studies or small-scale phase II clinical trials, and the indications for surgery were also uncertain, ranging from the most stringent standard of stage I $(12,13)$ or "peripheral small lesions" (14) to the broadest standard of "refer to the surgical principles of NSCLC" (15) or limited-period SCLC (Tx-4Nx-2M0) (16).

In view of the fact that these retrospective studies have defects, such as lack of suitable control groups $(6,7,17,18)$, indiscrimination in treating different stages of SCLC separately $(6,7,15)$, and the large time span of patients in these studies $(6,7,19,20)$, they do not provide high-quality evidence. Therefore, surgery with adjuvant chemotherapy is recommended for the treatment of clinical stage T1-2N0M0 SCLC in National Comprehensive Cancer Network guidelines (21). Furthermore, because of lack of surgical samples from patients with severe SCLC, to date, more studies are needed to evaluate the role of surgery in multidisciplinary treatment for patients with stage IIIA SCLC.

This study reviewed patients with clinical stage IIIA SCLC who underwent surgical resection with perioperative chemotherapy $(\mathrm{S}+\mathrm{C})$ or concurrent chemotherapy and radiotherapy (CRT) at Shanghai Pulmonary Hospital (SPH) from 2005 to 2015. We aimed to explore whether patients with clinical stage IIIA SCLC can benefit from surgery based on systemic chemotherapy.

We present the following article in accordance with the
STROBE reporting checklist (available at http://dx.doi. org/10.21037/jtd-20-3047).

\section{Methods}

\section{Study design}

The study was conducted in accordance with the Declaration of Helsinki (as revised in 2013). The study was approved by ethics board of K20-196Y and individual consent for this retrospective analysis was waived.

This study enrolled patients diagnosed with clinical stage IIIA SCLC at SPH from 2005 to 2015. For patients with stage I or stage II SCLC, the treatment plan at SPH was direct surgery after induction therapy, so few non-surgical patients were enrolled in the control group. Only patients with stage IIIA SCLC were informed of possible treatments without preferential advice, allowing patients to choose their next treatment plan.

The exclusion criteria for all patients were as follows: (I) a prior cancer diagnosis; (II) performance status (PS) $\geq 2$; (III) distant metastasis; (IV) treatment refusal; (V) requests to return to the local medical institution. The exclusion criteria for patients who underwent $\mathrm{S}+\mathrm{C}$ were as follows: (I) palliative surgery/a positive surgical margin; (II) death within 30 days of surgery. The exclusion criteria for patients who underwent CRT were as follows: (I) chemotherapy not completed according to the scheduled exposure plan; (II) radiotherapy not completed according to the scheduled exposure plan. Since pathological staging information of patients in the CRT group was not available, clinical staging criteria were used.

The diagnostic methods of SCLC include chest computed tomography (CT), brain magnetic resonance imaging (MRI), whole-body bone scans, ultrasonography, abdominal CT, and fiberoptic or electronic bronchoscopy. Positron emission tomography (PET)-CT was not mandatory for all patients. All patients underwent exfoliative cell examination of the sputum and bronchoscopic brush biopsy. Needle biopsies or endobronchial ultrasoundguided transbronchial needle aspiration (EBUS-TBNA) was performed in patients with peripheral or central lesions. All patients were confirmed the histological nature of SCLC before induction-chemotherapy.

Patients with a definite SCLC diagnosis first received two courses of platinum-based induction chemotherapy. After two courses of induction chemotherapy, patients were divided into two groups according to patients' personal 


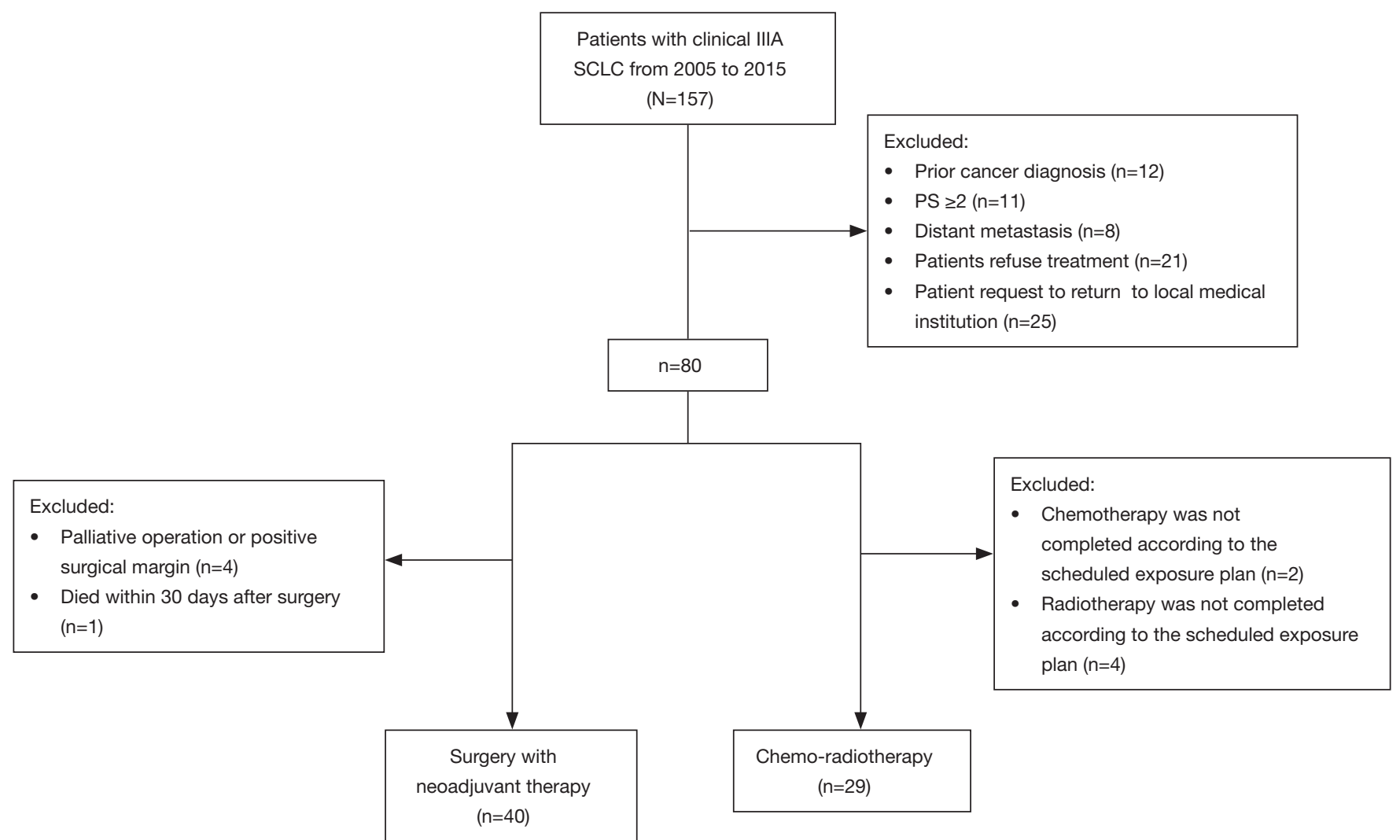

Figure 1 CONSORT diagram showing a schematic of study patient selection. SCLC, small cell lung cancer; PS, performance status.

willingness. The regimen separated at the end of second cycle of induction-chemotherapy. Patients who trend to receive surgery underwent surgery and subsequent adjuvant therapy and patients who refuse to underwent surgery received another 2-4 courses chemotherapy combined with radiotherapy. All confirmed SCLC patients received induction chemotherapy before surgery. Patients who were accidentally diagnosed with SCLC after surgery were not included in this study.

Survival was compared between patients who underwent CRT and those who underwent $\mathrm{S}+\mathrm{C}$. A survival analysis was performed using the Kaplan-Meier method. Multivariate Cox regression models were used to identify relevant variables affecting survival. All clinically significant variables were included in the model.

\section{Statistical analysis}

A two-sided $\mathrm{P}$ value of $<0.05$ was considered statistically significant. The statistical analysis was performed using SPSS 23.0 (SPSS Inc., Chicago, IL, USA), and survival curves were drawn using GraphPad Prism 6.0 (GraphPad Software, San Diego, CA, USA). The primary outcome was overall survival (OS). OS was defined as the time from diagnosis to death or final follow-up. Patients who were not deceased were censored on the date they were last known to be alive. Patient outcomes were measured up until June 30, 2017.

Comparisons of patient characteristics between patients with clinical stage IIIA SCLC who underwent $\mathrm{S}+\mathrm{C}$ and patients who underwent CRT were performed using the Student's $t$-test for continuous variables and Pearson's $\chi^{2}$ test or the Mann-Whitney $\mathrm{U}$ test for categorical variables.

\section{Results}

Between 2005 and 2015, 157 patients were diagnosed with clinical stage IIIA SCLC. Among them, 69 patients met the study inclusion criteria (Figure 1). Of these, surgery with neoadjuvant therapy was administered to 40 patients. Concurrent chemo-radiation was administered to 29 patients. 
Table 1 Chemotherapy scheme of cIIIA SCLC receiving concurrent chemotherapy and radiotherapy or systemic chemotherapy + surgery

\begin{tabular}{lcc}
\hline Chemotherapy & Concurrent chemo-radiotherapy $(\mathrm{N}=29)$ & Surgery with systemic chemotherapy $(\mathrm{N}=40)$ \\
\hline Etoposide + carboplatin & 13 & 22 \\
Etoposide + cisplatin & 13 & 11 \\
Irinotecan + carboplatin & 2 & 3 \\
Irinotecan + cisplatin & 1 & 4 \\
\hline
\end{tabular}

SCLC, small cell lung cancer.

All patients received systemic treatment consisting of cisplatin/carboplatin associated with etoposide/irinotecan. In detail, of the 40 patients in the $S+C$ group, 22 patients received etoposide plus carboplatin, 11 patients received etoposide plus cisplatin, 3 patients received irinotecan plus carboplatin, and 4 patients received irinotecan plus cisplatin. Of the 29 patients in the CRT group, 13 received etoposide plus carboplatin, 13 received etoposide plus cisplatin, 2 received irinotecan plus carboplatin, and 1 received irinotecan plus cisplatin (Table 1).

The clinical response to chemotherapy was assessable in all patients according to the Response Evaluation Criteria in Solid Tumors. In the $\mathrm{S}+\mathrm{C}$ group, 40 patients responded. Among them, 2 demonstrated complete responses (5.0\%), 33 demonstrated partial responses (82.5\%), and 5 had stable disease (12.5\%). In the CRT group, 29 patients responded. Among them, 17 demonstrated partial responses (58.6\%), 12 had stable disease (41.4\%), nobody had complete response. None of the patients interrupted chemotherapy because of toxicity.

For the type of surgery in the $\mathrm{S}+\mathrm{C}$ group, 22 patients underwent pneumonectomy (55.0\%), 14 patients underwent lobectomy (35.0\%), and 4 patients underwent bi-lobectomy $(10.0 \%)$. All of these patients underwent systematic lymph node dissection.

Nine patients in the $\mathrm{S}+\mathrm{C}$ group underwent percutaneous coronary intervention (PCI) $(22.5 \%)$, while 4 patients in the CRT group underwent PCI (13.8\%).

Compared with patients who underwent CRT, patients who underwent $\mathrm{S}+\mathrm{C}$ were associated with a younger age and a higher partial response to chemotherapy (Table 2).

Patients in the $\mathrm{S}+\mathrm{C}$ group were associated with a longer OS (median survival: 33.1 vs. 16.2 months, respectively; 2 -year OS: $44.2 \%$ vs. $14.9 \%$, respectively; log-rank: $\mathrm{P}=0.045$; Figure 2), compared with patients who underwent CRT. With a multivariate analysis, $\mathrm{S}+\mathrm{C}$ was associated with improved survival (hazard ratio: $0.374 ; 95 \%$ confidence interval: $0.173-0.808 ; \mathrm{P}=0.012 ;$ Table 3) compared with CRT.

\section{Discussion}

In this study, we evaluated the role of surgery in patients with clinical stage IIIA SCLC. For the two groups of patients who underwent the same courses of chemotherapy, surgery improved survival compared with radiotherapy, which indicated that with high-quality complete resection and chemotherapy, surgery may be an option in clinical stage IIIA SCLC after induction chemotherapy in selected patients.

To our knowledge, surgery is still controversial in patients with SCLC, particularly in patients with severe SCLC, as shown in two prospective randomized studies $(4,5)$. However, these two studies had some limitations. First, they lacked use of modern imaging staging methods, such as CT, PET-CT, and MRI, and modern mediastinal lymph node staging methods, such as EBUS-TBNA; thus, SCLC staging might be inaccurate. Second, both of these studies excluded patients with SCLC with peripherally isolated lesions without lymph node metastasis, for whom surgery would have had the most benefit. Third, patients in the surgery group did not achieve complete surgical resection. Specifically, some patients underwent open-chest exploration only or palliative resection. Fourth, neither of the studies used a platinum-based chemotherapy regimen, which might limit the effect of neoadjuvant chemotherapy and postoperative chemotherapy. Therefore, due to the difficulty in conducting prospective studies for patients with SCLC due to the limited number of patients with SCLC undergoing surgery, high-quality retrospective studies with comparisons between groups are essential to advance our understanding of the role of surgery for SCLC.

Rea et al. evaluated 53 patients with clinical stage III SCLC underwent surgery with perioperative neoadjuvant 
Table 2 Patients characteristics

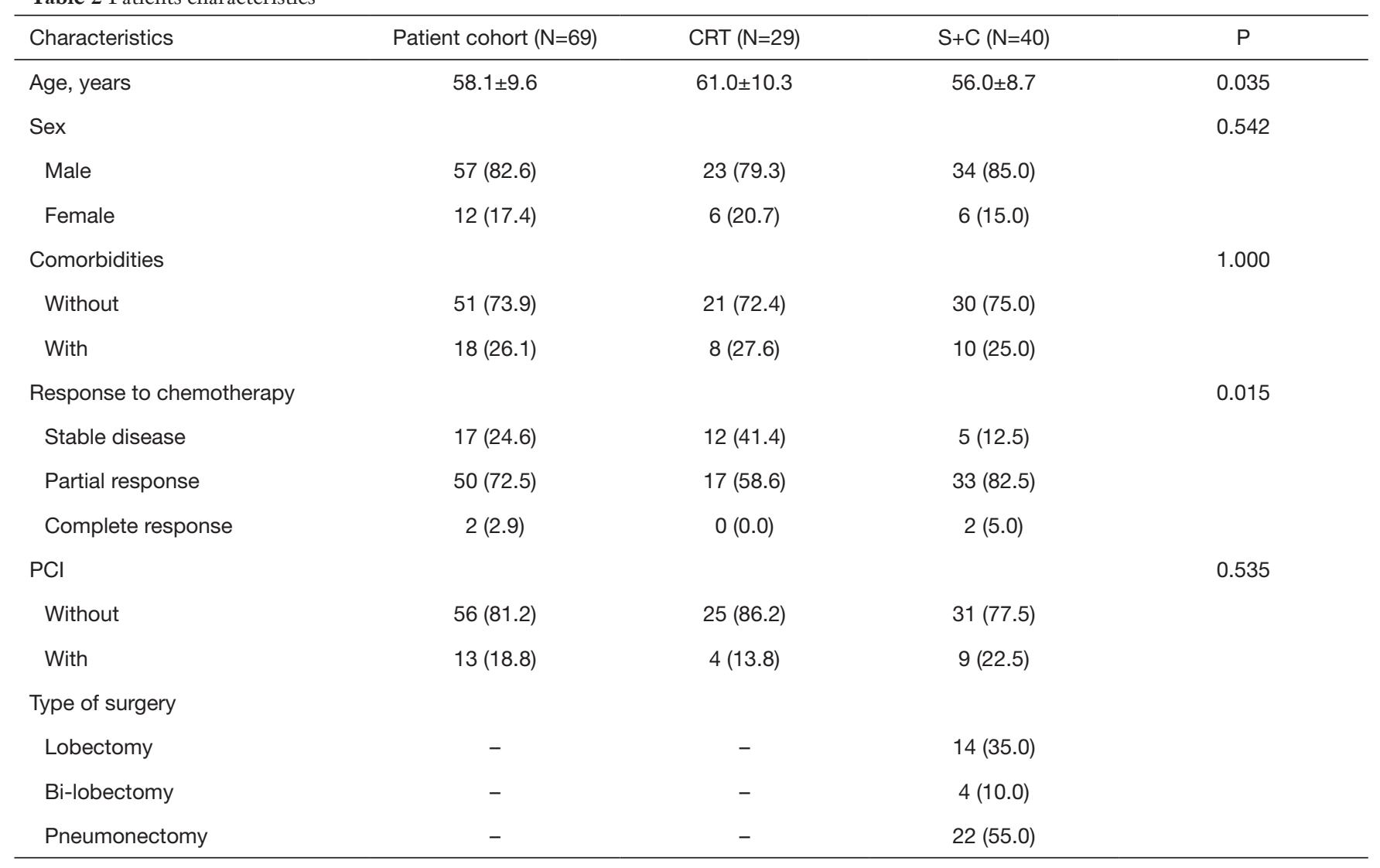

$\mathrm{PCl}$, prophylactic cranial irradiation; $\mathrm{S}+\mathrm{C}$, surgery with systemic chemotherapy; $\mathrm{CRT}$, concurrent chemo-radiotherapy.

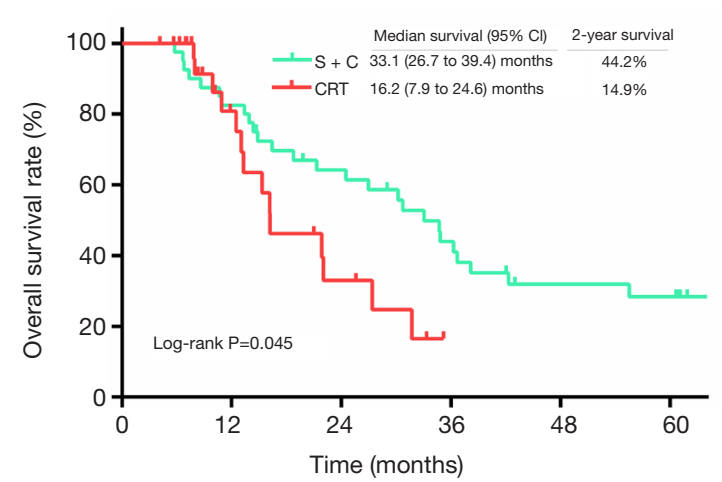

No. at risk

$\begin{array}{lrrrrrr}\mathrm{S}+\mathrm{C} & 40 & 33 & 23 & 15 & 9 & 8 \\ \mathrm{CRT} & 29 & 14 & 5 & 0 & 0 & 0\end{array}$

Figure 2 Overall survival of patients with clinical stage IIIA small cell lung cancer who underwent surgery with neoadjuvant and adjuvant chemotherapy $(\mathrm{S}+\mathrm{C})$ or concurrent chemo-radiotherapy (CRT). chemotherapy and postoperative adjuvant chemotherapy, and the 5-year survival rate was 32\% (18). Eberhardt et al. showed that multimodality treatment, including surgery, proved highly effective with $100 \%$ local control and remarkable long-term survival after complete resection, even in patients with locally advanced stage IIB/IIIA SCLC (17). Kawano et al. observed 19 patients with stage II-III SCLC and the 5-year survival rates were $63.5 \%$ and $42.7 \%$, respectively (22). Limited by lack of high-quality comparisons and limited chemotherapy data for patients undergoing surgery in databases such as the National Cancer Data Base (NCDB), these retrospective studies included patients over a large time span and patients with different staging criteria. The best treatment scheme for patients with stage IIIA SCLC is still unclear at present.

From current National Comprehensive Cancer Network guidelines (21), chemoradiotherapy with adjuvant PCI is 
Table 3 Multivariable analysis for patients with cIIIA SCLC

\begin{tabular}{|c|c|c|c|c|}
\hline Variable & $\mathrm{HR}$ & \multicolumn{2}{|c|}{$95 \% \mathrm{Cl}$} & $\mathrm{P}$ \\
\hline Age $\geq 60$ years & 0.754 & 0.354 & 1.604 & 0.463 \\
\hline Female vs. Male & 1.100 & 0.465 & 2.603 & 0.828 \\
\hline $\mathrm{PCl}$ & 0.518 & 0.209 & 1.284 & 0.156 \\
\hline \multicolumn{2}{|c|}{ Response to chemotherapy } & & & 0.112 \\
\hline PR vs. SD & 2.655 & 0.971 & 7.260 & 0.057 \\
\hline CR vs. SD & 6.189 & 0.589 & 64.994 & 0.129 \\
\hline Surgery & 0.374 & 0.173 & 0.808 & 0.012 \\
\hline
\end{tabular}

SCLC, small cell lung cancer; $\mathrm{Cl}$, confidence interval; PCI, prophylactic cranial irradiation; PR, partial response; SD, stable disease; CR, complete response.

suggested as an initial treatment for patients with limited stage IIIA SCLC. Stinchcombe et al. found that OS in older patients was 17.8 months, and OS in younger patients was 23.5 months (23). Hara et al. found that OS for patients with stage IIIA SCLC with CRT was 14 months (19). In our study, OS in the CRT group was 20 months, which is consistent with the literature $(19,23)$. The 30 years when chemotherapy was the first-line therapy for SCLC (24), as well as the EP (cisplatin + etoposide) and EC (carboplatin + etoposide) schemes, laid the foundation for chemotherapy for SCLC (25-27). However, the OS of patients with SCLC has now reached a bottleneck.

In our study, OS in the $\mathrm{S}+\mathrm{C}$ group was 32.5 months, which was significantly different compared with the CRT group (20.0 months, $\mathrm{P}=0.045)$. In 1995, Hara et al. found that OS in patients with stage IIIA SCLC who underwent surgery and chemotherapy was 16.5 months, while the non-surgery group had an OS of 14.0 months (19). Recently, with the application of adjuvant chemotherapy and the video-assisted thoracic surgery (VATS), the OS of patients with SCLC has prolonged. Zhang et al. showed a 5 -year survival rate of $36 \%$ (28). Wang et al. showed a 5 -year survival rate of $46.5 \%$ (29). The main purpose of surgical treatment is to remove the primary tumor, prevent recurrence, and reduce tumor burden. As part of the multidisciplinary treatment for SCLC, the role of surgery is becoming increasingly obvious.

There are several limitations in the present study. First, due to the inherent properties of retrospective studies, selection bias may exist; specifically, patients in the $\mathrm{S}+\mathrm{C}$ group were younger than patients in the CRT group, and the longer OS of patients in the $\mathrm{S}+\mathrm{C}$ group might have been caused by their younger age. Patients in the $\mathrm{S}+\mathrm{C}$ group also had a better response to induction chemotherapy. However, after adjusting for age and chemotherapy response, $\mathrm{S}+\mathrm{C}$ was still an independent factor associated with OS. Second, the setting of exclusion criteria in the CRT group should be more considerable because all the patient candidates to the treatment should be included, regardless of their completion, which could lead a longer OS in the CRT group. Third, since pathological staging in the medical treatment group is difficult to obtain, this study used clinical staging, which is less accurate than pathological staging. Fourth, this study selected patients from 2005 to 2015, with the last follow-up in 2017, because of the high loss ratio of follow-up after 2017. Further follow-up may help to get more accurate conclusions

\section{Conclusions}

Patients with clinical stage IIIA SCLC treated with surgical resection plus chemotherapy were associated with longer OS compared with those who underwent CRT. This conclusion needs further confirmation in prospective studies in the future.

\section{Acknowledgments}

Funding: This work was supported by the National Natural Science Foundation of China (No. 81972172), 
Shanghai Science and Technology Committee (No. 19XD1423200,18140903900), Shanghai Pulmonary Hospital (grant No. fkgg1801, fkcx1904), and the Clinical Research Plan of Shanghai Hospital Development Center (grant No. SHDC2020CR2020B, SHDC12018122).

\section{Footnote}

Reporting Checklist: The authors have completed the STROBE reporting checklist. Available at http://dx.doi. org/10.21037/jtd-20-3047

Data Sharing Statement: Available at http://dx.doi. org/10.21037/jtd-20-3047

Peer Review File: Available at http://dx.doi.org/10.21037/jtd20-3047

Conflicts of Interest: All authors have completed the ICMJE uniform disclosure form (available at http://dx.doi. org/10.21037/jtd-20-3047). The authors have no conflicts of interest to declare.

Ethical Statement: The authors are accountable for all aspects of the work in ensuring that questions related to the accuracy or integrity of any part of the work are appropriately investigated and resolved. The study was conducted in accordance with the Declaration of Helsinki (as revised in 2013). The study was approved by ethics board of K20-196Y and individual consent for this retrospective analysis was waived.

Open Access Statement: This is an Open Access article distributed in accordance with the Creative Commons Attribution-NonCommercial-NoDerivs 4.0 International License (CC BY-NC-ND 4.0), which permits the noncommercial replication and distribution of the article with the strict proviso that no changes or edits are made and the original work is properly cited (including links to both the formal publication through the relevant DOI and the license). See: https://creativecommons.org/licenses/by-nc-nd/4.0/.

\section{References}

1. Bernhardt EB, Jalal SI. Small Cell Lung Cancer. Cancer Treat Res 2016;170:301-22.

2. Globocan: Cancer Fact Sheets. Available online: http:// globocan.iarc.fr/tomorrow/graphic-bar. Accessed May
30, 2020.

3. Siegel RL, Miller KD, Jemal A. Cancer statistics, 2019. CA Cancer J Clin 2019;69:7-34.

4. Fox W, Scadding JG. Medical Research Council comparative trial of surgery and radiotherapy for primary treatment of small-celled or oat-celled carcinoma of bronchus. Ten-year follow-up. Lancet 1973;2:63-5.

5. Lad T, Piantadosi S, Thomas $\mathrm{P}$, et al. A prospective randomized trial to determine the benefit of surgical resection of residual disease following response of small cell lung cancer to combination chemotherapy. Chest 1994;106:320S-323S.

6. Lim E, Belcher E, Yap YK, et al. The role of surgery in the treatment of limited disease small cell lung cancer: time to reevaluate. J Thorac Oncol 2008;3:1267-71.

7. Inoue M, Miyoshi S, Yasumitsu T, et al. Surgical results for small cell lung cancer based on the new TNM staging system. Thoracic Surgery Study Group of Osaka University, Osaka, Japan. Ann Thorac Surg 2000;70:1615-9.

8. Nakamura H, Kato Y, Kato H. Outcome of surgery for small cell lung cancer -- response to induction chemotherapy predicts survival. Thorac Cardiovasc Surg 2004;52:206-10.

9. Clemente M, Soccal PM, Rochat T, et al. The role of surgery in early stage small cell lung cancer: should it be re-evaluated? Rev Med Suisse 2013;9:2170, 2172-4.

10. Meyer JA, Five-year survival in treated stage I and II small cell carcinoma of the lung. Ann Thorac Surg 1986;42:668-9.

11. Shepherd FA, Ginsberg RJ, Feld R, et al. Surgical treatment for limited small-cell lung cancer. The University of Toronto Lung Oncology Group experience. J Thorac Cardiovasc Surg 1991;101:385-93.

12. Yu JB, Blitzblau RC, Patel SC, et al. Surveillance, Epidemiology, and End Results (SEER) database analysis of microcystic adnexal carcinoma (sclerosing sweat duct carcinoma) of the skin. Am J Clin Oncol 2010;33:125-7.

13. Varlotto JM, Recht A, Flickinger JC, et al. Lobectomy leads to optimal survival in early-stage small cell lung cancer: a retrospective analysis. J Thorac Cardiovasc Surg 2011;142:538-46.

14. Rostad H, Naalsund A, Jacobsen R, et al. Small cell lung cancer in Norway. Should more patients have been offered surgical therapy? Eur J Cardiothorac Surg 2004;26:782-6.

15. Lüchtenborg M, Riaz SP, Lim E, et al. Survival of patients with small cell lung cancer undergoing lung resection in England, 1998-2009. Thorax 2014;69:269-73. 
16. Schreiber D, Rineer J, Weedon J, et al. Survival outcomes with the use of surgery in limited-stage small cell lung cancer: should its role be re-evaluated? Cancer 2010;116:1350-7.

17. Eberhardt W, Stamatis G, Stuschke M, et al. Prognostically orientated multimodality treatment including surgery for selected patients of small-cell lung cancer patients stages IB to IIIB: long-term results of a phase II trial. Br J Cancer 1999;81:1206-12.

18. Rea F, Callegaro D, Favaretto A, et al. Long term results of surgery and chemotherapy in small cell lung cancer. Eur J Cardiothorac Surg 1998;14:398-402.

19. Hara N, Ohta M, Ichinose $Y$, et al. Influence of surgical resection before and after chemotherapy on survival in small cell lung cancer. J Surg Oncol 1991;47:53-61.

20. Coolen L, Van den Eeckhout A, Deneffe G, et al. Surgical treatment of small cell lung cancer. Eur J Cardiothorac Surg 1995;9:59-64.

21. National Comprehensive Cancer Network: NCCN Clinical Practice Guidelines in Oncology: Small Cell Lung Cancer (version 2.2015). Fort Washington, PA: National comprehensive Cancer Network, 2015.

22. Kawano R, Tamaki K, Karita S, et al. Surgical treatment for clinical stage II and III small cell lung cancer patients. Kyobu Geka 2006;59:879-83; discussion 883-6.

23. Stinchcombe TE, Fan W, Schild SE, et al. A pooled analysis of individual patient data from National Clinical Trials Network clinical trials of concurrent

Cite this article as: Li S, Jin K, Pan Y, Wu C, Ren S, Jiang G, Zhang P. Role of surgery in a case-control study of patients with clinical stage IIIA small cell lung cancer. J Thorac Dis 2021;13(5):2738-2745. doi: 10.21037/jtd-20-3047 chemoradiotherapy for limited-stage small cell lung cancer in elderly patients versus younger patients. Cancer 2019;125:382-390.

24. McIllmurray MB, Bibby RJ, Taylor BE, et al. Etoposide compared with the combination of vincristine, doxorubicin, and cyclophosphamide in the treatment of small cell lung cancer. Thorax 1989;44:215-9.

25. Sundstrøm S, Bremnes RM, Kaasa S, et al. Cisplatin and etoposide regimen is superior to cyclophosphamide, epirubicin, and vincristine regimen in small-cell lung cancer: results from a randomized phase III trial with 5 years' follow-up. J Clin Oncol 2002;20:4665-72.

26. Rossi A, Di Maio M, Chiodini P, et al. Carboplatin- or cisplatin-based chemotherapy in first-line treatment of small-cell lung cancer: the COCIS meta-analysis of individual patient data. J Clin Oncol 2012;30:1692-8.

27. Fukuoka M, Furuse K, Saijo N, et al. Randomized trial of cyclophosphamide, doxorubicin, and vincristine versus cisplatin and etoposide versus alternation of these regimens in small-cell lung cancer. J Natl Cancer Inst 1991;83:855-61.

28. Zhang X, Sun Y, Huang J. Long-term results of small cell lung cancer treated by surgery combined with postoperative chemotherapy: a report of 65 patients. Zhonghua Zhong Liu Za Zhi 1997;19:137-9.

29. Wang Y, Sun YF, Chen KJ, et al. Surgical management of small intrahepatic lesions adjacent to the major vasculature. Zhonghua Wai Ke Za Zhi 2006;44:1631-3. 